\title{
Properties of lush spaces and applications to Banach spaces with numerical index 1
}

\author{
by \\ Kostyantyn Boyko (Kharkov), Vladimir Kadets (Kharkov), \\ Miguel Martín (Granada) and Javier Merí (Granada)
}

\begin{abstract}
The concept of lushness, introduced recently, is a Banach space property, which ensures that the space has numerical index 1. We prove that for Asplund spaces lushness is actually equivalent to having numerical index 1 . We prove that every separable Banach space containing an isomorphic copy of $c_{0}$ can be renormed equivalently to be lush, and thus to have numerical index 1 . The rest of the paper is devoted to the study of lushness just as a property of Banach spaces. We prove that lushness is separably determined, is stable under ultraproducts, and we characterize those spaces of the form $X=\left(\mathbb{R}^{n},\|\cdot\|\right)$ with absolute norm such that $X$-sum preserves lushness of summands, showing that this property is equivalent to lushness of $X$.
\end{abstract}

1. Introduction. Let us fix first some notations. All over the paper $X$ stands for a Banach space, $B_{X}$ and $S_{X}$ are, respectively, its closed unit ball and its unit sphere, $X^{*}$ is the dual space to $X$, and $L(X)$ is the Banach algebra of bounded linear operators on $X$. All linear spaces are over the field $\mathbb{K}$, which can be either the field $\mathbb{R}$ of reals or the field $\mathbb{C}$ of complex numbers. For a functional $x^{*} \in S_{X^{*}}$ and a positive number $\alpha$, the set of the form

$$
S\left(B_{X}, x^{*}, \alpha\right)=\left\{x \in B_{X}: \operatorname{Re} x^{*}(x)>1-\alpha\right\}
$$

is called a slice of the unit ball. If $A$ is a subset of $X$, we write $\operatorname{conv}(A)$ for the convex hull of $A$ and $\operatorname{aconv}(A)$ for the absolutely convex hull of $A$. Finally, we denote by $\operatorname{ext}(A)$ the set of extreme points of the convex subset $A \subseteq X$. Now the basic definition of our paper:

2000 Mathematics Subject Classification: Primary 46B20; Secondary 46B03, 46B04, 47A12.

Key words and phrases: numerical index, lush spaces, ultraproduct, Asplund spaces, renorming.

The work of the first-named author was partially supported by DAAD in the framework of Euler Fellowship. The second-named author conducted his work under the support of the Alexander-von-Humboldt fellowship. The third and fourth named authors were partially supported by Spanish MEC project no. MTM2006-04837 and Junta de Andalucía grants FQM-185 and P06-FQM-01438. 
Definition 1.1. A Banach space $X$ is said to be lush if for every $x, y$ $\in S_{X}$ and $\varepsilon>0$ there is a slice $S=S\left(B_{X}, x^{*}, \varepsilon\right) \subset B_{X}, x^{*} \in S_{X^{*}}$, such that $x \in S$ and $\operatorname{dist}(y, \operatorname{aconv}(S))<\varepsilon$.

Among the examples of lush spaces are the real or complex spaces $C(K)$ and $L_{1}(\mu)$ (see [5] or [9, §3] for a detailed account).

The concept of lushness was introduced recently in [5] as a geometrical property of a Banach space which ensures that the space has numerical index 1. The numerical index of a Banach space $X$ was introduced by G. Lumer in 1968 (see [6]) and it is the best constant of equivalence between the numerical radius and the usual norm of operators on $L(X)$. Concretely,

$$
\begin{aligned}
n(X) & =\inf \{v(T): T \in L(X),\|T\|=1\} \\
& =\max \{k \geq 0: k\|T\| \leq v(T) \forall T \in L(X)\} .
\end{aligned}
$$

Here, for $T \in L(X), v(T)$ is its numerical radius:

$$
v(T)=\sup \left\{\left|x^{*}(T(x))\right|: x \in S_{X}, x^{*} \in S_{X^{*}}, x^{*}(x)=1\right\} .
$$

Thus, a Banach space has numerical index 1 iff $v(T)=\|T\|$ for every $T \in L(X)$. It is clear from the definition that $0 \leq n(X) \leq 1$, these inequalities being best possible in the real case. In the complex case, it is a deep result that always $n(X) \geq 1 / e$. Classical references here are the monographs from the 1970's [2, 3]. The state-of-the-art on numerical indices may be found in the recent survey [9] and references therein.

The concept of lushness has proven to be a useful tool in the theory of numerical index of Banach spaces since in [5] it helped to construct an example showing that numerical index is not inherited in general by the dual space, a latent question in the theory from the beginning of the subject. Also, in [10] the lushness was applied to estimate the related concept of polynomial numerical index in some real spaces like $c_{0}$ or $\ell_{1}$.

One major difficulty when dealing with Banach spaces with numerical index 1 is that the definition involves operators, and one has to deal with all operators, since the analogous property with only compact operators (called the alternative Daugavet property) is known to be strictly weaker (see [15]). On the other hand, in the literature there are many isometric properties which are sufficient for a Banach space to have numerical index 1, lushness being the weakest (see $[9, \S 3]$ for a detailed account). In [14], it is shown that many of these properties are actually equivalent to numerical index 1 for Banach spaces with the Radon-Nikodým property. But this is not true for general Banach spaces (see [5, Example 3.4]). In this paper we prove that Asplund spaces with numerical index 1 are lush (Section 2).

Section 3 is devoted to renorming. We prove that every separable Banach space containing an isomorphic copy of $c_{0}$ can be equivalently renormed to be lush, and thus to have numerical index 1. Up to our knowledge, this is the 
first non-trivial sufficient condition for renorming with numerical index 1 . One can say that 1 is the only interesting value of the numerical index from the isomorphic point of view: in [7] it was proved that "many" Banach spaces (for instance, reflexive or separable spaces) can be equivalently renormed to have any possible value of the numerical index smaller than 1 (i.e. any number in $[0,1[$ in the real case and any number in $[1 / e, 1[$ in the complex case). On the other hand, some necessary conditions for a Banach space to be renormed with numerical index 1 were given in [12].

The rest of the paper is devoted to the study of lushness independently of its applications to numerical index. We show in Section 4 a reformulation of lushness which allows us to prove that it is separably determined and that it is inherited by ultraproducts. Finally, Section 5 is devoted to characterizing those spaces of the form $X=\left(\mathbb{R}^{n},\|\cdot\|\right)$ with absolute norm such that $X$-sum preserves lushness of summands (we show that this property is actually equivalent to lushness of $X$ ).

We have to stress out that the study of lush spaces is still in its "embryonal" faze, and that the number of open questions is much greater than that of results obtained.

2. Lushness and numerical index 1. In this section we give sufficient conditions for lushness which will be useful in the rest of the paper. Some notation is needed. Given a Banach space $X$, a subset $A \subset S_{X^{*}}$ is said to be norming for $X$ if

$$
\|x\|=\sup \left\{\left|a^{*}(x)\right|: a^{*} \in A\right\}
$$

for every $x \in X$. Given a completely regular Hausdorff topological space $\Omega$, we write $C_{b}(\Omega)$ to denote the Banach space of all $\mathbb{K}$-valued bounded continuous functions on $\Omega$, endowed with the supremum norm. Finally, we recall the fact that $X$ is Asplund if and only if $X^{*}$ has the Radon-Nikodým property.

Theorem 2.1. Let $X$ be a Banach space. Consider the following assertions.

(a) There is a completely regular Hausdorff topological space $\Omega$ and an isometric embedding $J: X \rightarrow C_{b}(\Omega)$ such that $\left|x^{* *}\left(J^{*}\left(\delta_{s}\right)\right)\right|=1$ for every $s \in \Omega$ and $x^{* *} \in \operatorname{ext}\left(B_{X^{* *}}\right)$.

(b) There is a norming set $A \subset B_{X^{*}}$ for $X$ such that $\left|x^{* *}\left(a^{*}\right)\right|=1$ for every $a^{*} \in A$ and $x^{* *} \in \operatorname{ext}\left(B_{X^{* *}}\right)$

(c) For each $x \in S_{X}$ and $\varepsilon>0$ there exists $x^{*} \in S_{X^{*}}$ such that

$$
x \in S=S\left(B_{X}, x^{*}, \varepsilon\right) \quad \text { and } \quad B_{X}=\overline{\operatorname{aconv}(S)} .
$$

(d) $X$ is lush.

(e) $n(X)=1$. 
Then $(\mathrm{a}) \Leftrightarrow(\mathrm{b}) \Rightarrow(\mathrm{c}) \Rightarrow(\mathrm{d}) \Rightarrow(\mathrm{e})$. If $X$ is an Asplund space, then all the assertions above are equivalent.

Proof. (a) $\Rightarrow$ (b). It suffices to show that $\left\{J^{*}\left(\delta_{s}\right): s \in \Omega\right\}$ is norming. To do so, for fixed $x \in X$ we observe that

$$
\sup _{s \in \Omega}\left[J^{*}\left(\delta_{s}\right)\right](x)=\sup _{s \in \Omega} \delta_{s}(J x)=\|J x\|=\|x\| .
$$

(b) $\Rightarrow$ (a). We consider the operator $J: X \rightarrow C_{b}(A)$ defined by

$$
[J x]\left(a^{*}\right)=a^{*}(x) \quad\left(x \in X, a^{*} \in A\right),
$$

which is isometric since the set $A \subset B_{X^{*}}$ is norming. Moreover, it is obvious from the definition of $J$ that $J^{*}\left(\delta_{a^{*}}\right)=a^{*}$ for every $a^{*} \in A$, and therefore

$$
\left|x^{* *}\left(J^{*}\left(\delta_{a^{*}}\right)\right)\right|=1
$$

for every $a^{*} \in A$ and every $x^{* *} \in \operatorname{ext}\left(B_{X^{* *}}\right)$.

(b) $\Rightarrow(\mathrm{c})$. Fix $\varepsilon>0$ and $x \in S_{X}$. We take $x^{*} \in A$ such that $\operatorname{Re} x^{*}(x)>$ $1-\varepsilon$, we define $S=S\left(B_{X}, x^{*}, \varepsilon\right)$, and we prove that $B_{X}=\overline{\operatorname{aconv}(S)}$. To do so, we define $\widetilde{S}=S\left(B_{X^{* *}}, x^{*}, \varepsilon\right)$ and we observe that the hypothesis gives us

$$
B_{X^{* *}}=\overline{\operatorname{aconv}(\widetilde{S})} w^{*} .
$$

On the other hand, it is clear that $\widetilde{S} \subset \bar{S}^{w^{*}}$, which tells us that

$$
\overline{\operatorname{aconv}(S)} w^{*}=\overline{\operatorname{aconv}(\widetilde{S})} w^{*}=B_{X^{* *}} .
$$

Finally, we deduce that

$$
B_{X}=B_{X^{* *}} \cap X=\overline{\operatorname{aconv}(S)} w^{*} \cap X=\overline{\operatorname{aconv}(S)}^{w}=\overline{\operatorname{aconv}(S)} .
$$

$(\mathrm{c}) \Rightarrow(\mathrm{d})$ is evident and $(\mathrm{d}) \Rightarrow(\mathrm{e})$ was proved in [5, Proposition 2.2].

Finally, the case when $X$ is Asplund follows from [5, Remark 3.5].

Let us point out that numerical index 1 and lushness are also equivalent for Banach spaces with the Radon-Nikodým property. This is an immediate consequence of [14, Theorem 1] and [5, Proposition 2.2].

REMARK 2.2. Let $X$ be a Banach space with the Radon-Nikodým property. Then $X$ is lush if and only if $n(X)=1$.

We now give two classes of spaces where Theorem 2.1 applies. The first class consists of preduals of $L_{1}(\mu)$ spaces. Indeed, it is clear that $\left|\int \varphi f d \mu\right|=1$ for every $f \in \operatorname{ext}\left(B_{L_{1}(\mu)}\right)$ and every $\varphi \in \operatorname{ext}\left(B_{L_{\infty}(\mu)}\right)$. Now, if $L_{1}(\mu)$ has a predual $X$, then the set $\operatorname{ext}\left(B_{L_{1}(\mu)}\right)$ is norming for $X$ and condition (b) of Theorem 2.1 applies.

EXAmPle 2.3. All preduals of any $L_{1}(\mu)$ space are lush. 
The second class of spaces to which Theorem 2.1 applies is the one of nicely embedded spaces in $C_{b}(\Omega)$ spaces. A Banach space $X$ is said to be nicely embedded in $C_{b}(\Omega)$ if there exists a linear isometry $J: X \rightarrow C_{b}(\Omega)$ such that for all $s \in \Omega$ the following properties are satisfied:

(N1) $\left\|J^{*} \delta_{s}\right\|=1$.

(N2) $\operatorname{span}\left(J^{*} \delta_{s}\right)$ is an $L$-summand in $X^{*}$.

This property was introduced in [18], where some examples can be found. It is immediate that nicely embedded spaces fulfill condition (a) in Theorem 2.1, so they are lush.

ExAmple 2.4. Any Banach space which nicely embeds into a $C_{b}(\Omega)$ space is lush.

We finish the section with a result concerning duals of Radon-Nikodým spaces. It is shown in [5, Proposition 4.1] that $n\left(X^{*}\right)=1$ if $X$ is a Banach space with the Radon-Nikodým property and $n(X)=1$. Actually, with the help of Theorem 2.1, it can be proved that $X^{*}$ is lush.

Proposition 2.5. Let $X$ be a Banach space with the Radon-Nikodym property and $n(X)=1$. Then for each $x^{*} \in S_{X^{*}}$ and $\varepsilon>0$ there exists $x^{* *} \in S_{X^{* *}}$ such that

$$
x^{*} \in S=S\left(B_{X^{*}}, x^{* *}, \varepsilon\right) \quad \text { and } \quad B_{X^{*}}=\overline{\operatorname{aconv}(S)} .
$$

In particular, $X^{*}$ is lush.

Proof. Following the proof of [5, Proposition 4.1], one finds that

$$
\left|x^{* * *}(a)\right|=1 \quad\left(x^{* * *} \in \operatorname{ext}\left(B_{X^{* * *}}\right), a \in A\right)
$$

where $A$ is the set of denting points of $B_{X}$ viewed as a subset of $B_{X^{* *}}$. Since $X$ has the Radon-Nikodým property, $A$ is a norming subset of $B_{X^{* *}}$ for $X^{*}$ and the result follows from Theorem 2.1.

3. Lush renormings. Our goal in this section is to prove that a separable Banach space containing an isomorphic copy of $c_{0}$ can be equivalently renormed to be lush (in particular, to have numerical index 1). We need two lemmata.

Lemma 3.1. Let $X$ be a separable Banach space containing an isometric copy of $c_{0}$. Then there is a biorthogonal system $\left\{\left(g_{n}, g_{n}^{*}\right)\right\} \subset B_{X} \times\left(12 B_{X^{*}}\right)$ such that

$$
\sup _{n \in \mathbb{N}}\left|g_{n}^{*}(x)\right| \geq \frac{1}{3}\|x\| \quad \text { for all } x \in X .
$$

Proof. Since a $c_{0}$-subspace of a separable space is 2-complemented (Sobczyk's theorem, see [1, Corollary 2.5.9] for instance), one can write $X$ as 
$c_{0} \oplus Y$ in such a way that for all $e \in c_{0}$ and $y \in Y$,

$$
\frac{1}{6}(\|e\|+\|y\|) \leq\|e+y\| \leq\|e\|+\|y\| .
$$

Denote by $\left\{e_{n}\right\}_{n \in \mathbb{N}}$ the canonical basis of $c_{0}$ and by $\left\{e_{n}^{*}\right\}_{n \in \mathbb{N}} \subset Y^{\perp} \subset X^{*}$ the corresponding coordinate functionals. By $(2),\left\|e_{n}^{*}\right\| \leq 6$ for every $n \in \mathbb{N}$. Now, we use the separability of $Y$ to choose a norming sequence $\left\{y_{n}^{*}\right\}_{n \in \mathbb{N}}$ $\subset S_{Y^{*}}$ with norming tails, that is,

$$
\sup _{n \geq m}\left|y_{n}^{*}(y)\right|=\|y\| \quad(y \in Y, m \in \mathbb{N}) .
$$

We write $\widetilde{y}_{n}^{*} \in c_{0}^{\perp} \subset X^{*}$ for the natural extensions of $y_{n}^{*}$ to the whole of $X$. Again, by (2), $\left\|\widetilde{y}_{n}^{*}\right\| \leq 6$. Let us show that $g_{n}=e_{n}, g_{n}^{*}=e_{n}^{*}+\widetilde{y}_{n}^{*}$ form the biorthogonal system we need. Indeed, consider an arbitrary $x=e+y \in X$, where $e \in c_{0}, y \in Y$. If $\|y\| \leq \frac{1}{3}\|x\|$, then $\|e\| \geq \frac{2}{3}\|x\|$ and

$$
\begin{aligned}
\sup _{n \in \mathbb{N}}\left|g_{n}^{*}(x)\right| & =\sup _{n \in \mathbb{N}}\left|e_{n}^{*}(e)+\widetilde{y}_{n}^{*}(y)\right| \\
& \geq \sup _{n \in \mathbb{N}}\left|e_{n}^{*}(e)\right|-\frac{1}{3}\|x\|=\|e\|-\frac{1}{3}\|x\| \geq \frac{1}{3}\|x\| .
\end{aligned}
$$

In the opposite case of $\|y\|>\frac{1}{3}\|x\|$, we select a sequence of indices $n_{1}<$ $n_{2}<\cdots$ such that $\left|\widetilde{y}_{n_{k}}^{*}(y)\right| \rightarrow\|y\|$. Then

$$
\begin{aligned}
\sup _{n \in \mathbb{N}}\left|g_{n}^{*}(x)\right| & \geq \limsup _{k \rightarrow \infty}\left|e_{n_{k}}^{*}(e)+\widetilde{y}_{n_{k}}^{*}(y)\right| \\
& =\limsup _{k \rightarrow \infty}\left|\widetilde{y}_{n_{k}}^{*}(y)\right|=\|y\|>\frac{1}{3}\|x\| .
\end{aligned}
$$

Lemma 3.2. Let $X$ be a separable Banach space containing an isomorphic copy of $c_{0}$. Then there is an isomorphic embedding $T: X \rightarrow \ell_{\infty}$ such that $T(X) \supset c_{0}$.

Proof. First, note that if $X$ contains an isomorphic copy of $c_{0}$, then $X$ can be renormed equivalently to have an isometric copy of $c_{0}$. Then choose $\left\{\left(g_{n}, g_{n}^{*}\right)\right\}_{n \in \mathbb{N}}$ as in Lemma 3.1 and define $T: X \rightarrow \ell_{\infty}$ as follows:

$$
T(x)=\left\{g_{n}^{*}(x)\right\}_{n \in \mathbb{N}} \in \ell_{\infty} \quad(x \in X) .
$$

The inequality (1) guarantees that

$$
\frac{1}{3}\|x\| \leq\|T(x)\| \leq 12\|x\| \quad \text { for all } x \in X,
$$

and the image of $g_{n}$ is the $n$th unit vector of $c_{0} \subset \ell_{\infty}$, so $T(X) \supset c_{0}$.

To finish our arguments, we need to use a class of lush spaces which was also introduced in the aforementioned paper [5], the so-called C-rich subspaces of $C(K)$.

Definition 3.3. Let $K$ be a compact Hausdorff space. A closed subspace $X$ of $C(K)$ is said to be $C$-rich if for every nonempty open subset $U$ of $K$ 
and every $\varepsilon>0$, there is a positive function $h$ of norm 1 with support inside $U$ such that the distance from $h$ to $X$ is less than $\varepsilon$.

Some examples of and remarks about C-rich subspaces will be needed.

REMARKS 3.4.

(a) Due to [5, Proposition 2.5], if $K$ is a perfect compact space, then every finite-codimensional subspace of $C(K)$ is $C$-rich and, in particular, lush.

(b) If one considers $\ell_{\infty}$ as $C(\beta \mathbb{N})$, then $c_{0}$ is $C$-rich in $\ell_{\infty}$. Indeed, this follows easily from the fact that $\mathbb{N}$ is a dense subset of $\beta \mathbb{N}$ consisting of isolated points.

(c) If $X \subset C(K)$ is $C$-rich, then every subspace $Y \subset C(K)$ containing $X$ is $C$-rich.

(d) In particular, every subspace of $\ell_{\infty}$ containing $c_{0}$ is $C$-rich.

(e) Let $K$ be an infinite compact set and $X$ be a Banach space such that it is $C$-rich in $C(K)$. Then $X$ contains an isomorphic copy of $c_{0}$. Indeed, choose a sequence of disjoint open sets $V_{n} \subset K$. Since $X$ is $C$-rich in $C(K)$, for $\varepsilon>0$ and $n \in \mathbb{N}$ we can find $f_{n} \in C(K)$ such that

$\operatorname{supp}\left(f_{n}\right) \subset V_{n}, \quad f_{n} \geq 0, \quad\left\|f_{n}\right\|=1, \quad$ and $\quad \operatorname{dist}\left(f_{n}, X\right) \leq \varepsilon / 2^{n}$.

The sequence $\left\{f_{n}\right\}$ is a $c_{0}$-basic sequence in $C(K)$, and a perturbation argument gives us a basic sequence in $X$ which is equivalent to $\left\{f_{n}\right\}$, and so it spans an isomorphic copy of $c_{0}$.

We are now able to state the main result of the section which characterizes isomorphically the separable Banach spaces containing $c_{0}$.

TheOREM 3.5. For a separable infinite-dimensional Banach space $X$, the following conditions are equivalent:

(i) $X$ contains an isomorphic copy of $c_{0}$.

(ii) $X$ is isomorphic to a $C$-rich subspace of $\ell_{\infty}=C(\beta \mathbb{N})$.

(iii) $X$ is isomorphic to a $C$-rich subspace of some $C(K)$.

Proof. (i) $\Rightarrow$ (ii). Lemma 3.2 tells us that there is an isomorphic embed$\operatorname{ding} T: X \rightarrow \ell_{\infty}$ such that $T(X) \supset c_{0}$. Thus, $T(X)$ is a C-rich subspace of $\ell_{\infty}$ by Remark 3.4.d and $X$ is isomorphic to $T(X)$. The implication (ii) $\Rightarrow($ iii) is evident and (iii) $\Rightarrow(\mathrm{i}$ ) is shown in Remark 3.4(e).

The following result is an evident consequence of the above theorem.

COROLlary 3.6. Every separable Banach space containing an isomorphic copy of $c_{0}$ can be equivalently renormed to be lush and, in particular, to have numerical index 1.

As an easy consequence we obtain the following. 
Corollary 3.7. Any closed subspace of $c_{0}$ can be renormed to be lush and, in particular, to have numerical index 1.

Proof. Let $X$ be a closed subspace of $c_{0}$. If $X$ is finite-dimensional, the result is clear. Otherwise, $X$ contains an isomorphic copy of $c_{0}[1$, Proposition 2.1.1] and the result follows from the above corollary.

As far as we know, these are the first non-trivial sufficient conditions for renorming with numerical index 1 appearing in the literature, and they give a partial positive answer to Problem 21 of [9].

We finish this section with an example showing that the answer to Problem 22 of [9] (whether any Banach space such that $X^{*}$ contains an isomorphic copy of $\ell_{1}$ is renormable with numerical index 1 ) is negative.

EXAMPLE 3.8. There is a Banach space $Y$ such that $Y^{*}$ is isomorphic to $\ell_{1}$ but $Y$ does not admit an equivalent norm with numerical index 1 . Indeed, consider the real space $Y$ given in [4] such that $Y^{*}$ is isomorphic to $\ell_{1}$ and $Y$ has the Radon-Nikodým property. Then $Y$ is an infinite-dimensional real Banach space having the Radon-Nikodým property and it is also Asplund, so [12, Corollary 4] shows that it does not admit an equivalent norm with numerical index 1 .

4. A reformulation of lushness. In this section we prove a useful reformulation of lushness which does not use slices. We use it to reduce lushness of a non-separable space to the lushness of "sufficiently many" separable subspaces and to study lushness of ultraproducts.

THEOREM 4.1. Let $X$ be a Banach space and let $G \subset S_{X^{*}}$ be a norming rounded subset for $X$. Then the following are equivalent:

(i) $X$ is lush.

(ii) $)_{\mathbb{R}}$ In the real case: for every $x, y \in S_{X}$ and $\varepsilon>0$, there are $\lambda_{1}, \lambda_{2} \geq 0$ with $\lambda_{1}+\lambda_{2}=1$ and $x_{1}, x_{2} \in B_{X}$ such that

$$
\left\|x+x_{1}+x_{2}\right\|>3-\varepsilon
$$

and

$$
\left\|y-\left(\lambda_{1} x_{1}-\lambda_{2} x_{2}\right)\right\|<\varepsilon .
$$

(ii) $\mathbb{C}$ In the complex case: For every $x, y \in S_{X}, n \in \mathbb{N}$ and $\varepsilon>0$, there exist $\lambda_{1}, \ldots, \lambda_{n} \geq 0$ with $\sum_{k=1}^{n} \lambda_{k}=1$ and $x_{1}, \ldots, x_{n} \in B_{X}$ such that

$$
\left\|x+\sum_{k=1}^{n} x_{k}\right\|>n+1-\varepsilon
$$


and

$$
\left\|y-\sum_{k=1}^{n} \lambda_{k} \exp \left(\frac{2 \pi i k}{n}\right) x_{k}\right\|<\varepsilon+\frac{2 \pi}{n} .
$$

(iii) For every $x, y \in S_{X}$ and $\varepsilon>0$ there is $x^{*} \in G$ such that $x \in S=$ $S\left(B_{X}, x^{*}, \varepsilon\right)$ and $\operatorname{dist}(y, \operatorname{aconv}(S))<\varepsilon$ (i.e. $x^{*}$ in the definition of lushness can be chosen from $G$ ).

Proof. Let us start with the complex case.

(i) $\Rightarrow$ (ii). Fix $x, y \in S_{X}, n \in \mathbb{N}$ and $\varepsilon>0$. Since $X$ is a lush space, we may find $x^{*} \in S_{X^{*}}, \mu_{j} \in[0,1], \theta_{j} \in[0,2 \pi]$ and $y_{j} \in S\left(B_{X}, x^{*}, \varepsilon / n\right)$, $j=1, \ldots, N$, with $\sum_{j=1}^{N} \mu_{j}=1$, satisfying

$$
\operatorname{Re} x^{*}(x)>1-\varepsilon / n \text { and }\left\|y-\sum_{j=1}^{N} \mu_{j} \exp \left(i \theta_{j}\right) y_{j}\right\|<\varepsilon \text {. }
$$

Since the points $\{2 \pi k / n: k=1, \ldots, n\}$ form an $2 \pi / n$-net of $[0,2 \pi]$ we can split $\{1, \ldots, N\}$ into disjoint sets $A_{k}, k=1, \ldots, n$, in such a way that

$$
\left|\theta_{j}-2 \pi k / n\right| \leq 2 \pi / n \quad \text { for every } j \in A_{k} .
$$

Let us show that one can take

$$
\lambda_{k}=\sum_{j \in A_{k}} \mu_{j} \quad \text { and } \quad x_{k}=\frac{1}{\lambda_{k}} \sum_{j \in A_{k}} \mu_{j} y_{j} \quad \text { if } A_{k} \neq \emptyset
$$

and

$$
\lambda_{k}=0 \quad \text { and arbitrary } \quad x_{k} \in S\left(B_{X}, x^{*}, \varepsilon / n\right) \quad \text { if } A_{k}=\emptyset .
$$

Indeed, it is clear that $x_{k} \in S\left(B_{X}, x^{*}, \varepsilon / n\right)$, which ensures (4). The remaining condition (5) follows from (6) and (7):

$$
\begin{aligned}
\left\|y-\sum_{k=1}^{n} \lambda_{k} \exp \left(\frac{2 \pi i k}{n}\right) x_{k}\right\| & =\left\|y-\sum_{k: A_{k} \neq \emptyset} \sum_{j \in A_{k}} \mu_{j} \exp \left(\frac{2 \pi i k}{n}\right) y_{j}\right\| \\
& \leq\left\|y-\sum_{k: A_{k} \neq \emptyset} \sum_{j \in A_{k}} \mu_{j} \exp \left(i \theta_{j}\right) y_{j}\right\|+\frac{2 \pi}{n} \\
& =\left\|y-\sum_{j=1}^{N} \mu_{j} \exp \left(i \theta_{j}\right) y_{j}\right\|+\frac{2 \pi}{n}<\varepsilon+\frac{2 \pi}{n} .
\end{aligned}
$$

(ii) $\Rightarrow$ (iii). For given $x, y \in S_{X}$ and $\varepsilon_{1}>0$, we apply (ii) with $\varepsilon=\varepsilon_{1} / 2$ and $n$ large enough to ensure that $2 \pi / n<\varepsilon_{1} / 2$. We get $\lambda_{1}, \ldots, \lambda_{n} \geq 0$ with $\sum_{k=1}^{n} \lambda_{k}=1$ and $x_{1}, \ldots, x_{n} \in B_{X}$ satisfying (4) and (5). The first of these conditions ensures the existence of $x^{*} \in G$ such that $x$ and all the $x_{k}$ belong to the slice $S\left(B_{X}, x^{*}, \varepsilon\right) \subset S\left(B_{X}, x^{*}, \varepsilon_{1}\right)$. The second condition shows that $\operatorname{dist}\left(y, \operatorname{aconv}\left(S\left(B_{X}, x^{*}, \varepsilon_{1}\right)\right)\right)<\varepsilon_{1}$, which proves lushness of $X$. 
The last implication (iii) $\Rightarrow$ (i) is evident.

In the real case, $S_{\mathbb{R}}$ consists just of two points 1 and -1 , and the parameters $n$ and $\exp (2 \pi i k / n)$ disappear from the statement. The proof follows the same lines as in the complex case.

The following is an application of the above characterization.

Theorem 4.2. For a Banach space $X$ the following two conditions are equivalent:

(i) $X$ is lush.

(ii) Every separable subspace $E \subset X$ is contained in a separable lush subspace $Y \subset X$.

Proof. The implication (ii) $\Rightarrow($ i) is immediate from the definition of lushness.

Let us prove only the more bulky complex case of (i) $\Rightarrow\left(\right.$ ii). Let $\left(k_{n}, j_{n}\right)$ be the standard "triangle" enumeration of all pairs of naturals. Note that, under this enumeration, $k_{n} \leq n$ and $j_{n} \leq n$ for all $n \in \mathbb{N}$. Let us construct recurrently a sequence of separable subspaces $E_{1} \subset E_{2} \subset \cdots$, dense sequences $A_{m}=\{a(m, r): r \in \mathbb{N}\} \subset S_{E_{m}}$ and $x_{m}, y_{m} \in S_{X}$ as follows: for $m=1$ we take $E_{1}=E$, let $A_{1}=\{a(1, r): r \in \mathbb{N}\}$ be a dense sequence in $S_{E_{1}}$ with every element repeated infinitely many times, and set $x_{1}=a\left(k_{k_{1}}, j_{k_{1}}\right)=a(1,1), y_{1}=a\left(k_{j_{1}}, j_{j_{1}}\right)=a(1,1)$. If $E_{m}$ and $A_{m}$ are already constructed, we consider $x_{m}=a\left(k_{k_{m}}, j_{k_{m}}\right), y_{m}=a\left(k_{j_{m}}, j_{j_{m}}\right)$ and apply Theorem 4.1 (ii) to $x_{m}, y_{m}$ with $\varepsilon_{m}=1 / m$ for $n=2, \ldots, m$. We get $\lambda_{m, n, 1}, \ldots, \lambda_{m, n, n} \geq 0$ with $\sum_{k=1}^{n} \lambda_{m, n, k}=1$ and $x_{m, n, 1}, \ldots, x_{m, n, n} \in B_{X}$ such that

$$
\left\|x_{m}+\sum_{k=1}^{n} x_{m, n, k}\right\|>n+1-\varepsilon_{m}
$$

and

$$
\left\|y_{m}-\sum_{k=1}^{n} \lambda_{m, n, k} \exp \left(\frac{2 \pi i k}{n}\right) x_{m, n, k}\right\|<\varepsilon_{m}+\frac{2 \pi}{n}
$$

for $n=2, \ldots, m$. Define $E_{m+1}$ as the linear span of $E_{m}$ and of all the vectors $x_{m, n, s}, n=2, \ldots, m, s=1, \ldots, n$. Let $A_{m+1}=\{a(m+1, r): r \in \mathbb{N}\}$ be a dense sequence in $S_{E_{m+1}}$ with every element repeated infinitely many times. Put $Y=\overline{\bigcup_{m \in \mathbb{N}} E_{m}}$. Under this construction $Y$ is separable, contains $E$, and the pair $\left(x_{m}, y_{m}\right)$ runs over all possible values of $(a(r, s), a(t, u))$, $r, s, t, u \in \mathbb{N}$. Indeed, for the last assertion we observe that the map $m \mapsto$ $\left(k_{m}, j_{m}\right)$ is bijective from $\mathbb{N}$ to $\mathbb{N} \times \mathbb{N}$, so the map $(m, n) \mapsto\left(\left(k_{m}, j_{m}\right),\left(k_{n}, j_{n}\right)\right)$ is bijective from $\mathbb{N} \times \mathbb{N}$ to $((\mathbb{N} \times \mathbb{N}) \times(\mathbb{N} \times \mathbb{N}))$. Thus, the composition of these two maps $m \mapsto\left(\left(k_{k_{m}}, j_{k_{m}}\right),\left(k_{j_{m}}, j_{j_{m}}\right)\right)$ is bijective from $\mathbb{N}$ to 
$((\mathbb{N} \times \mathbb{N}) \times(\mathbb{N} \times \mathbb{N}))$ and so

$$
\begin{aligned}
\left\{\left(x_{m}, y_{m}\right): m \in \mathbb{N}\right\} & \left.=\left\{\left(a\left(k_{k_{m}}, j_{k_{m}}\right), a\left(k_{j_{m}}, j_{j_{m}}\right)\right)\right): m \in \mathbb{N}\right\} \\
& =\{(a(i, j), a(k, l)):(i, j, k, l) \in \mathbb{N} \times \mathbb{N} \times \mathbb{N} \times \mathbb{N}\} .
\end{aligned}
$$

Therefore, $\left\{\left(x_{m}, y_{m}\right): m \in \mathbb{N}\right\}$ is dense in $S_{Y} \times S_{Y}$ and has every element repeated infinitely many times. Due to our construction, this means that item (ii) of Theorem 4.1 holds for any $x, y$ from the fixed dense subset $\left\{\left(x_{m}, y_{m}\right): m \in \mathbb{N}\right\}$ of $S_{Y} \times S_{Y}$ for all $n \in \mathbb{N}$ and with arbitrarily small epsilons. This means that $Y$ is lush.

Let us comment that, since for Asplund spaces lushness is equivalent to numerical index 1 (Theorem 2.1), the above result improves [13, Teorema 3], where the following is proved. Let $X$ be an Asplund, weakly countably determined space with numerical index 1 . Then, for every separable subspace $Y$ of $X$, there is another separable subspace $Z$ of $X$ containing $Y$ and such that $n(Z)=1$.

We finish the section with an application of Theorem 4.1 to ultraproducts. Let us recall the notion of (Banach) ultraproducts [8]. Let $\mathcal{U}$ be a free ultrafilter on $\mathbb{N}$, and let $\left\{X_{n}\right\}_{n \in \mathbb{N}}$ be a sequence of Banach spaces. We can consider the $\ell_{\infty}$-sum of the family, $\left[\bigoplus_{n \in \mathbb{N}} X_{n}\right]_{\ell_{\infty}}$, together with its closed subspace

$$
N_{\mathcal{U}}=\left\{\left\{x_{n}\right\}_{n \in \mathbb{N}} \in\left[\bigoplus_{n \in \mathbb{N}} X_{n}\right]_{\ell_{\infty}}: \lim _{\mathcal{U}}\left\|x_{n}\right\|=0\right\} .
$$

The quotient space $\left(X_{n}\right)_{\mathcal{U}}=\left[\bigoplus_{n \in \mathbb{N}} X_{n}\right]_{\ell_{\infty}} / N_{\mathcal{U}}$ is called the ultraproduct of the family $\left\{X_{n}\right\}_{n \in \mathbb{N}}$ relative to the ultrafilter $\mathcal{U}$. Let $\left(x_{n}\right)_{\mathcal{U}}$ stand for the element of $\left(X_{n}\right)_{\mathcal{U}}$ containing a given family $\left\{x_{n}\right\} \in\left[\bigoplus_{n \in \mathbb{N}} X_{n}\right]_{\ell_{\infty}}$. It is easy to check that

$$
\left\|\left(x_{n}\right) \mathcal{U}\right\|=\lim _{\mathcal{U}}\left\|x_{n}\right\| .
$$

If all the $X_{n}$ are equal to the same Banach space $X$, the ultraproduct of the family is called the $\mathcal{U}$-ultrapower of $X$ and is usually denoted by $X_{\mathcal{U}}$.

Our next goal is to show that lushness is inherited by ultraproducts. Actually, the following stronger version of lushness will be obtained for the ultraproducts.

Definition 4.3. A complex Banach space $X$ is said to be ultra-lush if for every $x, y \in S_{X}$ and $n \in \mathbb{N}$ there exist $\lambda_{1}, \ldots, \lambda_{n} \geq 0$ with $\sum_{k=1}^{n} \lambda_{k}=1$ and $x_{1}, \ldots, x_{n} \in B_{X}$ such that

$$
\left\|x+\sum_{k=1}^{n} x_{k}\right\|=n+1 \quad \text { and }\left\|y-\sum_{k=1}^{n} \lambda_{k} \exp \left(\frac{2 \pi i k}{n}\right) x_{k}\right\| \leq \frac{2 \pi}{n} .
$$

A real Banach space $X$ is said to be ultra-lush if for every $x, y \in S_{X}$, there 
exist $\lambda_{1}, \lambda_{2} \geq 0$ with $\lambda_{1}+\lambda_{2}=1$ and $x_{1}, x_{2} \in S_{X}$ such that

$$
\left\|x+x_{1}+x_{2}\right\|=3 \text { and } y=\lambda_{1} x_{1}-\lambda_{2} x_{2} .
$$

As straightforward applications of Theorem 4.1 and the definition of ultraproducts, we get the following results.

Corollary 4.4. Let $\left\{X_{n}\right\}_{n \in \mathbb{N}}$ be a sequence of lush spaces and let $\mathcal{U}$ be a free ultrafilter on $\mathbb{N}$. Then $E=\left(X_{n}\right)_{\mathcal{U}}$ is ultra-lush. Moreover, the ultraproduct of any sequence of Banach spaces is ultra-lush whenever it is lush.

Corollary 4.5. Let $X$ be a Banach space and $\mathcal{U}$ be a free ultrafilter on $\mathbb{N}$. Then the ultrapower $E=X_{\mathcal{U}}$ is lush if and only if $X$ is lush.

5. Unconditional sums of lush spaces. A norm $\|\cdot\|_{\mathrm{a}}$ on $\mathbb{R}^{n}$ is said to be an absolute norm if

$$
\left\|\left(a_{1}, \ldots, a_{n}\right)\right\|_{\mathrm{a}}=\left\|\left(\left|a_{1}\right|, \ldots,\left|a_{n}\right|\right)\right\|_{\mathrm{a}} \quad\left(a_{1}, \ldots, a_{n} \in \mathbb{R}\right)
$$

and $\|(1,0, \ldots, 0)\|_{\mathrm{a}}=\cdots=\|(0, \ldots, 0,1)\|_{\mathrm{a}}=1$. If $E=\left(\mathbb{R}^{n},\|\cdot\|_{\mathrm{a}}\right)$ is a space with an absolute norm and $X_{1}, \ldots, X_{n}$ are Banach spaces, we write $X=\left[X_{1} \oplus \cdots \oplus X_{n}\right]_{E}$ to denote the $E$-direct sum of $X_{1}, \ldots, X_{n}$, that is, $X=X_{1} \oplus \cdots \oplus X_{n}$ endowed with the norm

$$
\left\|\left(x_{1}, \ldots, x_{n}\right)\right\|=\left\|\left(\left\|x_{1}\right\|, \ldots,\left\|x_{n}\right\|\right)\right\|_{\mathrm{a}} .
$$

We will use the fact that absolute norms are non-decreasing and continuous in each variable. For background, we refer the reader to [3, §21]. Easy examples of absolute norms are the $\ell_{p}$-norms for $1 \leq p \leq \infty$ leading to the $\ell_{p}$-direct sums of Banach spaces.

Definition 5.1. Let $E=\left(\mathbb{R}^{n},\|\cdot\|\right)$ be a Banach space with an absolute norm. We say that $E$ respects lushness if for every collection $X_{1}, \ldots, X_{n}$ of lush spaces their $E$-direct sum $X=\left[X_{1} \oplus \cdots \oplus X_{n}\right]_{E}$ is lush.

Our goal in this section is to characterize those absolute norms which respect lushness.

TheOREM 5.2. A space $E=\left(\mathbb{R}^{n},\|\cdot\|\right)$ respects lushness if and only if it is lush itself.

Proof. The "only if" part is evident: if $E$ respects lushness, we can take all $X_{k}=\mathbb{R}$ and get lushness of $E=\left[X_{1} \oplus \cdots \oplus X_{n}\right]_{E}$.

Let us prove the "if" part. Let $X_{1}, \ldots, X_{n}$ be lush spaces with $X=$ $\left[X_{1} \oplus \cdots \oplus X_{n}\right]_{E}$. First of all, let us remark that if all $X_{k}$ are finitedimensional, then by [17, Theorem 3.1] lushness is equivalent to the following property:

$$
\left|x^{*}(x)\right|=1 \quad\left(\text { for every } x \in \operatorname{ext}\left(B_{X}\right) \text { and every } x^{*} \in \operatorname{ext}\left(B_{X^{*}}\right)\right) .
$$


This property can be easily verified taking into account that

$$
\operatorname{ext}\left(B_{X}\right)=\left\{\left(a_{1} x_{1}, \ldots, a_{n} x_{n}\right):\left(a_{1}, \ldots, a_{n}\right) \in \operatorname{ext}\left(B_{E}\right), x_{k} \in \operatorname{ext}\left(B_{X_{k}}\right)\right\},
$$

the analogous description for $\operatorname{ext}\left(B_{X^{*}}\right)$, and the fact that the sets of extreme points in $B_{E}$ and in $B_{E^{*}}$ admit rotation in each variable.

Now, let us turn to the general case where some of $X_{k}$ are infinitedimensional. By a small perturbation argument, it is sufficient to prove the property of the definition of lushness for a fixed $\varepsilon>0$ and $x, y \in S_{X}$, $x=\left(x_{1}, \ldots, x_{n}\right), y=\left(y_{1}, \ldots, y_{n}\right)$ with non-zero $x_{k}, y_{k}$. Set

$$
\alpha_{k}=\left\|x_{k}\right\|, \quad \beta_{k}=\left\|y_{k}\right\|, \quad \widetilde{x}_{k}=\frac{x_{k}}{\alpha_{k}}, \quad \widetilde{y}_{k}=\frac{y_{k}}{\beta_{k}}, \quad \delta=\frac{\varepsilon}{n+3} .
$$

By lushness of $X_{k}$, there is $x_{k}^{*} \in S_{X_{k}^{*}}$ such that

$$
\widetilde{x}_{k} \in S_{k}=S\left(B_{X_{k}}, x_{k}^{*}, \delta\right) \text { and } \operatorname{dist}\left(\widetilde{y}_{k}, \operatorname{aconv}\left(S_{k}\right)\right)<\delta .
$$

This means that there are a finite number of elements $u_{k, 1}, \ldots, u_{k, m_{k}} \in S_{k}$ and a finite number of scalars $\lambda_{k, 1}, \ldots, \lambda_{k, m_{k}}$ such that $\sum_{j=1}^{m_{k}}\left|\lambda_{k, j}\right|=1$, and

$$
\left\|\widetilde{y}_{k}-\sum_{j=1}^{m_{k}} \lambda_{k, j} u_{k, j}\right\|<\delta .
$$

We embed $X_{k}$ isometrically into some infinite-dimensional space $\widetilde{X}_{k}$ (if $X_{k}$ is infinite-dimensional, then put $\widetilde{X}_{k}=X_{k}$ ), set $\widetilde{X}=\left[\widetilde{X}_{1} \oplus \cdots \oplus \widetilde{X}_{n}\right]_{E}$ and select linearly independent vectors $e_{k, 1}, \ldots, e_{k, m_{k}} \in S_{\widetilde{X}_{k}}$ so that

$$
\sum_{j=1}^{m_{k}}\left\|e_{k, j}-u_{k, j}\right\|<\delta .
$$

We consider $Y_{k}=\operatorname{span}\left\{e_{k, j}: j=1, \ldots, m_{k}\right\}$ and introduce a new norm $p_{k}$ on $Y_{k}$ in such a way that $B_{\left(Y_{k}, p_{k}\right)}=\operatorname{aconv}\left(\left\{e_{k, j}: j=1, \ldots, m_{k}\right\}\right)$. Note that

$$
p_{k}(w) \geq\|w\| \quad \text { for every } w \in Y_{k} .
$$

Observe also that the linear map $g_{k}:\left(Y_{k}, p_{k}\right) \rightarrow X_{k}$ with $g_{k}\left(e_{k, j}\right)=u_{k, j}$ satisfies

$$
g_{k}\left(B_{\left(Y_{k}, p_{k}\right)}\right)=\operatorname{aconv}\left(\left\{u_{k, j}: j=1, \ldots, m_{k}\right\}\right)
$$

and

$$
g_{k}\left(\operatorname{conv}\left(\left\{e_{k, j}: j=1, \ldots, m_{k}\right\}\right)\right)=\operatorname{conv}\left(\left\{u_{k, j}: j=1, \ldots, m_{k}\right\}\right) .
$$

Moreover, thanks to (9), $\left\|w_{k}-g_{k}\left(w_{k}\right)\right\|<\delta$ for every $w_{k} \in B_{\left(Y_{k}, p_{k}\right)}$.

We introduce one more auxiliary space $(Y, p)=\left[\left(Y_{1}, p_{1}\right) \oplus \cdots \oplus\left(Y_{n}, p_{n}\right)\right]_{E}$. As proved before, since $\left(Y_{k}, p_{k}\right)$ is isometric to $\ell_{1}^{\left(m_{k}\right)}$ for every $k, Y$ is a finite-dimensional lush space, and therefore $n(Y)=1$, implying by [11, Corollary 3.7] that $Y$ is a CL-space. This means, by definition, that $B_{Y}$ is the absolutely convex hull of every maximal convex subset (maximal face) of $S_{Y}$. 
For every $w=\left(w_{1}, \ldots, w_{n}\right) \in B_{(Y, p)}$ we set $g(w)=\left(g_{1}\left(w_{1}\right), \ldots, g_{n}\left(w_{n}\right)\right)$. Then

$$
\|w-g(w)\|_{\widetilde{X}}<\|(\delta, \ldots, \delta)\|_{E} \leq n \delta
$$

and

$$
\|g(w)\|_{X} \leq p(w) .
$$

Consider $u=\left(\alpha_{1} \widetilde{z}_{1}, \ldots, \alpha_{n} \widetilde{z}_{n}\right)$, where

$$
\widetilde{z}_{k}=\frac{1}{m_{k}} \sum_{j=1}^{m_{k}} e_{k, j},
$$

and $v=\left(\beta_{1} \widetilde{v}_{1}, \ldots \beta_{n} \widetilde{v}_{n}\right)$, where

$$
\widetilde{v}_{k}=\sum_{j=1}^{m_{k}} \lambda_{k, j} e_{k, j}
$$

Then $u, v \in S_{Y}$, so there is an $f=\left(f_{1}, \ldots, f_{n}\right) \in S_{(Y, p)^{*}}$ such that $f(u)=1$ and the absolute convex hull of the face $F=\left\{w \in S_{(Y, p)}: \operatorname{Re} f(w)=1\right\}$ contains $v$.

We define $\gamma_{k}=p_{k}^{*}\left(f_{k}\right)$ and $D=\left\{k: \gamma_{k} \neq 0\right\}$. Then we have $\gamma=$ $\left(\gamma_{1}, \ldots, \gamma_{n}\right) \in S_{E^{*}}, \alpha=\left(\alpha_{1}, \ldots, \alpha_{n}\right) \in S_{E}$ and

$$
1=\operatorname{Re} f(u)=\sum_{k=1}^{n} \alpha_{k} \operatorname{Re} f_{k}\left(\widetilde{z}_{k}\right) \leq \sum_{k=1}^{n} \alpha_{k} \gamma_{k} \leq 1 .
$$

Therefore,

$$
\sum_{k=1}^{n} \alpha_{k} \gamma_{k}=1 \quad \text { and } \quad f_{k}\left(\widetilde{z}_{k}\right)=\gamma_{k} \quad(k=1, \ldots, n) .
$$

So, in particular, $f_{k} / \gamma_{k} \in S_{\left(Y_{k}^{*}, p_{k}^{*}\right)}$ is a supporting functional of the point $\widetilde{z}_{k}$ for every $k \in D$, i.e. $f_{k}\left(e_{k, j}\right)=\gamma_{k}$ for all $k, j$.

Finally, let us introduce $x^{*}=\left(\gamma_{1} x_{1}^{*}, \ldots, \gamma_{n} x_{n}^{*}\right) \in S_{X^{*}}$ and let us prove that the slice $S=S\left(B_{X}, x^{*}, \varepsilon\right)$ is the one we need, namely that $x \in S$ and $\operatorname{dist}(y, \operatorname{aconv}(S))<\varepsilon$.

The inclusion $x \in S$ is simple:

$$
\operatorname{Re} x^{*}(x)=\sum_{k=1}^{n} \alpha_{k} \gamma_{k} \operatorname{Re} x_{k}^{*}\left(\widetilde{x}_{k}\right)>\sum_{k=1}^{n} \alpha_{k} \gamma_{k}(1-\delta)=1-\delta .
$$

To estimate the distance from $y$ to $\operatorname{aconv}(S)$ we need the following claim:

Claim. $g(w) \in S$ for every $w=\left(w_{1}, \ldots, w_{n}\right) \in F$.

Proof of the claim. First of all, according to (12), $\|g(w)\| \leq 1$, so the only thing we need to prove is the estimate

$$
\operatorname{Re} x^{*}(g(w))>1-\varepsilon .
$$


Set $\phi_{k}=p_{k}\left(w_{k}\right)$. By the definition of $F$, we have

$$
1=f(w)=\sum_{k=1}^{n} \operatorname{Re} f_{k}\left(w_{k}\right) \leq \sum_{k=1}^{n} \gamma_{k} \phi_{k} \leq 1,
$$

and therefore

$$
\sum_{k=1}^{n} \gamma_{k} \phi_{k}=1 \quad \text { and } \quad f_{k}\left(w_{k}\right)=\gamma_{k} \phi_{k} \quad(k=1, \ldots, n) .
$$

This means that $w_{k} / \phi_{k} \in \operatorname{conv}\left(\left\{e_{k, j}: j=1, \ldots, m_{k}\right\}\right)$ for every $k$ such that $\gamma_{k} \phi_{k} \neq 0$, and so $g_{k}\left(w_{k}\right) / \phi_{k} \in \operatorname{conv}\left(\left\{u_{k, j}: j=1, \ldots, m_{k}\right\}\right) \subset S_{k}$. Therefore

$$
\operatorname{Re} x^{*}(g(w))=\sum_{k=1}^{n} \gamma_{k} \operatorname{Re} x_{k}^{*}\left(g_{k}\left(w_{k}\right)\right) \geq \sum_{k=1}^{n} \gamma_{k} \phi_{k}(1-\delta)>1-\varepsilon,
$$

which gives the claim.

Now, let $u_{1}, \ldots, u_{m}$ be elements of the face $F$ and let $\lambda_{1}, \ldots, \lambda_{m}$ be scalars such that $\sum_{j=1}^{m}\left|\lambda_{j}\right|=1$ and $v=\sum_{j=1}^{m} \lambda_{j} u_{j}$. Thanks to the claim, $g\left(u_{j}\right) \in S$. Therefore

$$
\operatorname{dist}(y, \operatorname{aconv}(S)) \leq\left\|y-\sum_{j=1}^{m} \lambda_{j} g\left(u_{j}\right)\right\| \leq\|y-v\|+\left\|v-\sum_{j=1}^{m} \lambda_{j} g\left(u_{j}\right)\right\| .
$$

We continue the estimation using the definition of $v$ and the inequalities (8) and (11):

$$
\leq 2 \delta\left\|\left(\beta_{1}, \ldots, \beta_{n}\right)\right\|_{E}+\sum_{j=1}^{m}\left|\lambda_{j}\right|\left\|u_{j}-g\left(u_{j}\right)\right\| \leq(n+2) \delta<\varepsilon .
$$

This completes the proof of the theorem.

Although the above theorem only deals with finite sums of lush spaces, one can deduce from it the lushness of some infinite sums. Given an arbitrary family $\left\{X_{i}: i \in I\right\}$ of Banach spaces, we denote by $\left[\bigoplus_{i \in I} X_{i}\right]_{c_{0}}$ (resp. $\left.\left[\bigoplus_{i \in I} X_{i}\right]_{\ell_{1}},\left[\bigoplus_{i \in I} X_{i}\right]_{\ell_{\infty}}\right)$ the $c_{0}$-sum (resp. $\ell_{1}$-sum, $\ell_{\infty}$-sum) of the family.

Proposition 5.3. Let $\left\{X_{i}: i \in I\right\}$ be a family of lush spaces. Then the $c_{0}-, \ell_{1}-$ and $\ell_{\infty}$-sums of the family are also lush.

Proof. Let us start with the easier cases $c_{0^{-}}$or $\ell_{1}$-sum of the family. In these two cases, we consider the family of all finite sums $\left(c_{0}\right.$ or $\ell_{1}$ respectively) of elements of the family $\left\{X_{i}: i \in I\right\}$, viewed as subspaces of the whole $c_{0}$ - or $\ell_{1}$-sum. Then Theorem 5.2 tells us that this is a family of lush subspaces which is filtered and whose union is dense. The result now follows straightforwardly from the definition of lushness.

For the $\ell_{\infty}$-sum of the family the above argument does not apply, but it is not difficult to give a direct proof. Let $X$ denote the $\ell_{\infty}$-sum of the family, 
let $\left(x_{i}\right),\left(y_{i}\right)$ be elements in the unit sphere of $X$ and let $\varepsilon>0$ be fixed. By the definition of $X$, there is $i_{0} \in I$ such that $\left\|x_{i_{0}}\right\|>1-\varepsilon$. Since $X_{i_{0}}$ is lush, we may find $x_{i_{0}}^{*} \in S_{X_{i_{0}}^{*}}$ such that, writing $S_{i_{0}}=S\left(B_{X_{i_{0}}}, x_{i_{0}}^{*}, \varepsilon\right)$, we have

$$
x_{i_{0}} \in S_{i_{0}} \quad \text { and } \operatorname{dist}\left(y_{i_{0}}, \operatorname{aconv}\left(S_{i_{0}}\right)\right)<\varepsilon .
$$

Now, defining $\widetilde{x}^{*} \in S_{X^{*}}$ as

$$
\widetilde{x}^{*}\left(\left(z_{i}\right)\right)=x_{i_{0}}^{*}\left(z_{i_{0}}\right) \quad\left(\left(z_{i}\right) \in X\right),
$$

and writing $\widetilde{S}=S\left(B_{X}, \widetilde{x}^{*}, \varepsilon\right)$, we clearly have

$$
\left(x_{i}\right) \in \widetilde{S} \text { and } \operatorname{dist}\left(\left(y_{i}\right), \operatorname{aconv}(\widetilde{S})\right)<\varepsilon \text {. }
$$

Let us comment that the analogue of Proposition 5.3 for Banach spaces with numerical index 1 was given in [16, Corollary 4]. On the other hand, we do not know of any result analogous to Theorem 5.2 for Banach spaces with numerical index 1 .

Acknowledgements. The authors thank the anonymous referee for multiple stylistical improvements.

\section{References}

[1] F. Albiac and N. J. Kalton, Topics in Banach Space Theory, Grad. Texts in Math. 233, Springer, New York, 2006.

[2] F. F. Bonsall and J. Duncan, Numerical Ranges of Operators on Normed Spaces and of Elements of Normed Algebras, London Math. Soc. Lecture Note Ser. 2, Cambridge Univ. Press, 1971.

[3] —, -, Numerical Ranges II, London Math. Soc. Lecture Note Ser. 10, Cambridge Univ. Press, 1973.

[4] J. Bourgain and F. Delbaen, A class of special $\mathcal{L}_{\infty}$ spaces, Acta Math. 145 (1980), $155-176$.

[5] K. Boyko, V. Kadets, M. Martín and D. Werner, Numerical index of Banach spaces and duality, Math. Proc. Cambridge Philos. Soc. 142 (2007), 93-102.

[6] J. Duncan, C. McGregor, J. Pryce, and A. White, The numerical index of a normed space, J. London Math. Soc. 2 (1970), 481-488.

[7] C. Finet, M. Martín, and R. Payá, Numerical index and renorming, Proc. Amer. Math. Soc. 131 (2003), 871-877.

[8] S. Heinrich, Ultraproducts in Banach space theory, J. Reine Angew. Math. 313 (1980), 72-104.

[9] V. Kadets, M. Martín, and R. Payá, Recent progress and open questions on the numerical index of Banach spaces, Rev. R. Acad. Cien. Ser. A Mat. 100 (2006), $155-182$.

[10] S. G. Kim, M. Martín, and J. Merí, On the polynomial numerical index of the real spaces $c_{0}, l_{1}$ and $l_{\infty}$, J. Math. Anal. Appl. 337 (2008), 98-106.

[11] Å. Lima, Intersection properties of balls in spaces of compact operators, Ann. Inst. Fourier (Grenoble) 28 (1978), 35-65.

[12] G. López, M. Martín, and R. Payá, Real Banach spaces with numerical index 1, Bull. London Math. Soc. 31 (1999), 207-212. 
[13] M. Martín, Numerical index and subspaces, in: Proc. of the Meeting of Andalusian Mathematicians, Vol. II (Sevilla, 2000), Colecc. Abierta 52, Univ. Sevilla Secr. Publ., Seville, 2001, 641-648 (in Spanish).

[14] —, Banach spaces having the Radon-Nikodým property and numerical index 1, Proc. Amer. Math. Soc. 131 (2003), 3407-3410.

[15] M. Martín and T. Oikhberg, An alternative Daugavet property, J. Math. Anal. Appl. 294 (2004), 158-180.

[16] M. Martín and R. Payá, Numerical index of vector-valued function spaces, Studia Math. 142 (2000), 269-280.

[17] C. M. McGregor, Finite dimensional normed linear spaces with numerical index 1, J. London Math. Soc. 3 (1971), 717-721.

[18] D. Werner, The Daugavet equation for operators on function spaces, J. Funct. Anal. 143 (1997), 117-128.

Department of Mechanics and Mathematics

Kharkov National University

Pl. Svobody 4

61077 Kharkov, Ukraine

E-mail: k_boyko@ukr.net

vova1kadets@yahoo.com
Departamento de Análisis Matemático Facultad de Ciencias Universidad de Granada 18071 Granada, Spain E-mail: mmartins@ugr.es jmeri@ugr.es

Received September 17, 2007

Revised version June 17, 2008 